\title{
Bricks for different houses
}

DOI:

10.1038/nrn2247

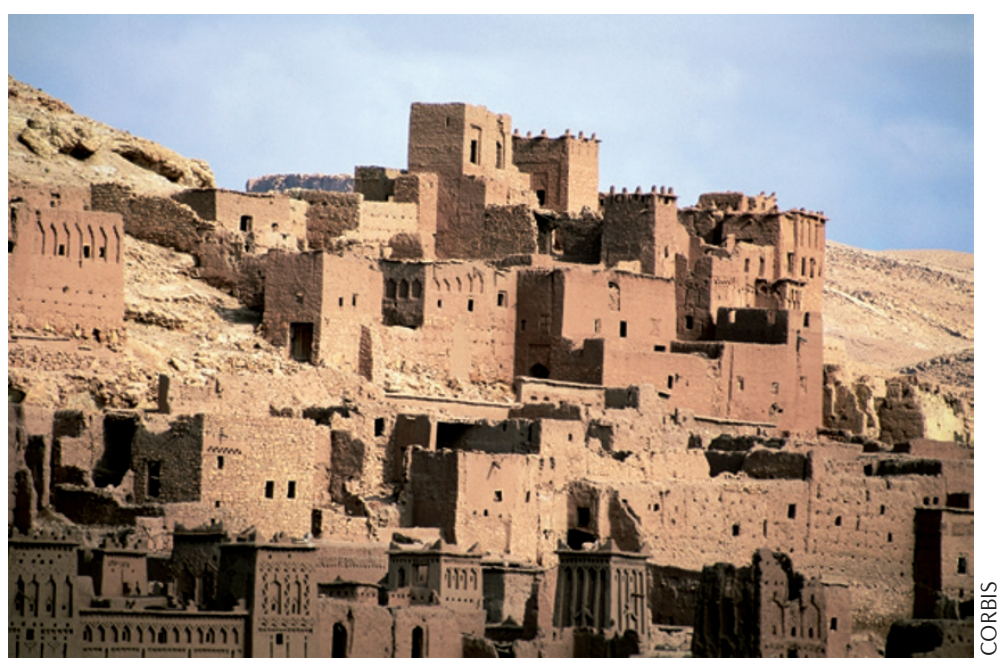

The supply of membrane components to growth cones by the secretory pathway is a requirement for the outgrowth of axons and dendrites alike. However, it is unclear whether differences in the secretory pathway account for some of the differences in axonal and dendritic development. Jan and colleagues now demonstrate that membrane supply is differentially regulated in dendritic and axonal growth and that Golgi outposts in the dendrites have a vital role in the development of the dendritic tree.

The authors screened for Drosophila melanogaster dendritic arbor reduction (dar) mutants and identified — among others - dar3, a gene that has been implicated in ER-to-Golgi trafficking. Interestingly, mutations in the mammalian dar3 homologue, SAR1, also reduced dendritic length in cultured hippocampal neurons, pointing to an evolutionarily conserved role for this protein. Furthermore, the rate of membrane supply to dendrites, but not to axons, was impaired in SAR1 mutants, suggesting that dendritic and axonal growth rely on different membrane supply mechanisms.

Jan and colleagues noticed that mutations in dar3 and SAR1 not only reduced the overall dendritic length in D. melanogaster neurons and cultured hippocampal neurons, respectively, but also changed the appearance of the normally compact Golgi structures in the soma and dendrites (Golgi outposts) such that they were more diffuse, suggesting that dar3 and SAR1 are required for normal Golgi morphology.

The role of Golgi outposts was further investigated in D. melanogaster. Damaging Golgi outposts with intense laser illumination resulted in excessive stability of the distal dendrities, which was in line with the author's proposal that Golgi outposts promote the growth of distal dendrites. Disruption of the function of

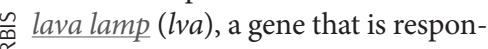
sible for the transport of Golgi along microtubules, altered the distribution of Golgi outposts, but not of the somatic Golgi: distal outposts were smaller, whereas proximal outposts were larger. This was paralleled by reduced branching of distal dendrites and increased branching of proximal dendrites.

These findings demonstrate that Golgi outposts are required for dendritic outgrowth and that different membrane supply mechanisms underlie the differentially regulated development of axons and dendrites. Claudia Wiedemann

ORIGINAL RESEARCH PAPER Ye, B. et al. Growing dendrites and axons differ in their reliance on the secretory pathway. Cell 130 , 717-729 (2007) 